\title{
Instituto Nacional de Vigilancia de Medicamentos y Alimentos: una muerte anunciada
}

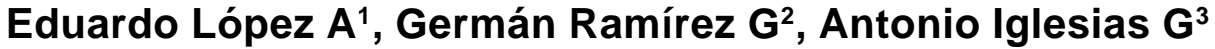 \\ ${ }^{1}$ QF Catedrático. Programa de Bacteriología y Laboratorio Clínico. Facultad de Ciencias de la salud. Universidad Colegio Mayor de \\ Cundinamarca. Ex Jefe División de Control de Medicamentos INS. \\ ${ }^{2}$ MD Profesor titular, Facultad de Medicina de la Universidad del Rosario. Ex jefe Sección de Control Biológico INS. \\ ${ }^{3}$ MD Profesor Titular. Facultad de Medicina de la Universidad Nacional. Ex Director Instituto Nacional de Salud. \\ Recibido: 01-08-04 / Aceptado: 12-09-04
}

\begin{abstract}
Resumen
El control de medicamentos, aguas y productos biológicos se ha realizado en el país en forma continua desde 1919, como función o bajo la supervisión del Ministerio que con diferentes nombres ha estado encargado de los asuntos de Higiene, Salud, Trabajo y Seguridad Social. Esta actividad se ejecutaba en forma dispersa por intermedio de varias instituciones publicas o privadas lo que llevó, en la última década del siglo pasado a considerar la creación de una institución de orden nacional que tuviera estas funciones y las ejerciera con mayor eficacia. Así nació el Instituto Nacional de Vigilancia de Medicamentos y Alimentos (Invima) creado por la Ley 100 del 23 de diciembre de 1993, el cual comenzó a ejercer sus funciones a partir de febrero de 1995. Diez años después de su creación, el gobierno ha manifestado sus intenciones de acabar con él y trasladar sus funciones a la Superintendencia Nacional de Salud. Esta decisión conllevaría un enorme retroceso en el sistema de vigilancia y control, en detrimento del aseguramiento de la calidad de los medicamentos y alimentos, como elementos fundamentales en el bienestar y la salud del pueblo colombiano.
\end{abstract}

Palabra clave: INVIMA, vigilancia y control, medicamentos, productos biológicos.

\section{Abstract \\ National Institute of Medicines and Foods Alertness: an announced death}

The control of medications, waters and biological products ace to function of State one eats making in continuous form from 1919 when the Congress of Colombia creó the Bacteriological and Chemical Laboratory, like to dependence of the National Direction of Hygiene calling it "Official Laboratory of Hygiene." This function of Surveillance and Control is more efficient when in 1947, the government buys the Laboratory "Samper Martínez" to his founders who carried out these controls to his biological products that elaborated in that laboratory. A recount is made the Ministry of Health that has had several names then already in 1920 called himself later Ministry working Hygiene and Social Prevention and 80 years of how it is denominated Ministry of the Social Protection. This Ministry has always worried to exercise the surveillance and control to the medications and allowances, activity that has executed in dispersed form to inclination several institutions 
what took to consider the creation of an institution of national order that had these functions and it exercised them with more effectiveness. It was born this way the National Institute of Surveillance of Medications and Allowances (INVIMA) created by the Law 100 of December 231993 and it began to exercise their functions starting from February of 1995. Ten years after their creation the Government has manifested his intentions of passing his functions to the National Superintendence of Health. Lastly it is questioned if the Government's attitude is due to a weakness in the operation of this institution or to that has not completed with the functions that were assigned him. Situation that he/she would go in the detriment of the insurance of the quality of the medications and allowances, as fundamental elements in the well-being and the consumer's health.

Key words: INVIMA, alertness and control, medicines, biological products.

\section{Introducción}

Este artículo tiene por objeto dar a conocer la situación que existía a nivel nacional en el sector de la salud sobre la vigilancia y control de los alimentos, bebidas y medicamentos como bienes de consumo esenciales para el desarrollo y conservación de la vida, antes de la creación del Instituto Nacional de Vigilancia de Medicamentos y Alimentos (Invima) y alertar sobre los peligros, riesgos y consecuencias para el país ante su desaparición.

Con el ánimo de llegar a éste propósito se mencionan las principales instituciones del sector salud que tradicionalmente tuvieron alguna acción en el proceso de control de los productos que hoy día son controlados y vigilados por el Invima de acuerdo con sus funciones.

Reseña. Históricamente la función de vigilancia y control de los alimentos y bebidas ha sido desempeñada por el Ministerio de Salud, actualmente denominado Ministerio de la Protección Social, exceptuándose al inicio del siglo pasado, cuando la tenía el Ministerio de Trabajo, Higiene y Prevención Social que ejercía las funciones relacionadas con la salud pública en el país.

El control de medicamentos, aguas y productos biológicos en Colombia se inicia 1919 cuando el Congreso de la República crea el "Laboratorio Oficial de Higiene", dependencia de la Dirección Nacional de Higiene, asignada por repartición al Ministerio de
Trabajo, Higiene y Prevención Social, con funciones de laboratorio bacteriológico y químico (1).

Tres décadas después, el Ministerio de Higiene adopta como laboratorio de referencia para ejercer la función de vigilancia y control de los alimentos y medicamentos al Instituto Nacional de Higiene “Samper Martínez", que desde 1947 tuvo a su cargo funciones como:

- Producción de sueros, vacunas y demás productos biológicos o químicos, destinados a la lucha contra las enfermedades comunicables o producidas por mordeduras de animales venenosos.

- El estudio y control de sustancias medicamentosas $\mathrm{y}$ alimenticias que se producían en laboratorios o establecimientos particulares.

- La vigilancia, reglamentación y examen de productos biológicos para usos terapéuticos o profilácticos, como sueros vacunas, etc., preparados en laboratorios oficiales o particulares.

- El análisis de sustancias o productos farmacéuticos, que le fueran enviados para otorgamiento de licencia y venta en el territorio de la república. Así como la preparación de la vacuna antirrábica. En 1962 el instituto "Samper Martínez" fue integrado al Instituto Nacional de Salud, conservando hasta finales de 1995 sus funciones de laboratorio de referencia del Ministerio de Salud en el área de producción y de la vigilancia y control de los alimentos y medicamentos. 
Así pues, el Instituto Nacional de Salud tenía por aquellos años una doble función: de un lado, elaborar los productos biológicos que el Ministerio de Salud requería para las campañas de prevención y tratamiento de enfermedades prevenibles; y por el otro, realizar sus respectivos controles para el aseguramiento de la calidad conforme a las Normas vigentes. Además, se encargaba de realizar los análisis a los alimentos, bebidas y medicamentos para la obtención del registro sanitario y el control de calidad en todas las líneas de comercialización de estos productos.

En conclusión, gran parte de las acciones de vigilancia y control que el Ministerio de Salud ejercía sobre la calidad de los medicamentos y alimentos, las ejecutaba a través del INS y se realizaban por medio de pruebas de laboratorio que comprendían análisis físicos, químicos, microbiológicos, biológicos y toxicológicos, de acuerdo con los parámetros establecidos por el Ministerio del ramo.

Cumplimiento de estándares internacionales: la Organización Mundial de la Salud (OMS) reconoce la importancia de las pruebas de laboratorio en el control, cuando manifiesta que "...el laboratorio de análisis es la piedra angular de todo programa tendiente a garantizar la inocuidad de los alimentos; considerarlo un simple servicio de apoyo constituye un grave aunque muy extendido error" (2).

Tradicionalmente este concepto tenido en cuenta por las autoridades sanitarias colombianas encargadas de la vigilancia y control de los productos de consumo, en especial de los alimentos, bebidas y medicamentos, pues la certeza de que un producto esté libre de contaminación o cumpla las normas de calidad, sólo la puede ofrecer -en casi la totalidad de los casos- una prueba analítica producida por el laboratorio. El concepto emitido por el laboratorio de análisis del INS era un requisito indispensable y determinante para la aceptación o rechazo de un producto por parte del Ministerio de Salud.

www.unicolmayor.edu.co
Sin embargo, el Instituto Nacional de Salud ejercía funciones tanto en el área de la producción, como en la de control de los productos, condición que le hacía "juez y parte", lo que le ubicaba en una situación insostenible, abiertamente inconveniente. La Oficina Sanitaria Panamericana, a través de sus asesores en Colombia, les manifestó a los funcionarios del INS los inconvenientes de esta dualidad.

Independiente de esta situación institucional, el sistema de vigilancia y control de los medicamentos y alimentos existente en el país por 1980, por parte del sector oficial, era disperso y poco efectivo.

El INVIMA, solución a problemas evidentes: el Ministerio de Salud disponía de una red de laboratorios de análisis de alimentos y de medicamentos a nivel nacional, que ejercían en forma aislada las funciones de control de calidad y qué, además, trabajaban sin coordinación y con ineficiencia; situación que se había puesto en evidencia en 1978, cuando se presentó en el país una intoxicación con alcohol metílico debido al uso de alcohol antiséptico como medio para controlar la temperatura de los niños, y éste producto, "alcohol antiséptico", de libre venta y distribución a nivel nacional, presentaba una adulteración con metanol en el $42.5 \%$ de las muestras analizadas en la sección de análisis del Instituto $\mathrm{Na}-$ cional de Salud. El empleo de alcohol antiséptico adulterado, causó numerosas muertes, en especial en población infantil. Esta situación fue altamente divulgada a través de los medios de comunicación de su época (3).

En 1991 el Ministerio de Salud dentro de su plan de acción 1991-1994 recomienda la creación de un sistema de control de calidad para los medicamentos e insumos de salud, alimentos y demás productos industriales que puedan ocasionar riesgos, de tal manera que se garantice su eficacia y baja toxicidad. El sistema incluirá entre otras las siguientes acciones:

- Inspección de fábricas y vigilancia farmacológica de los productos comercializados

- Servicios de laboratorio para realizar los análisis químicos y físicos de productos. 
Las Directivas del INS desarrollaron una estrategia para establecer los contactos a nivel nacional con los gremios, asociaciones, universidades e instituciones de salud del área de los medicamentos y alimentos con el propósito de informarlos y concertar con ellos su aporte en conocimientos para llevar a feliz término la creación de la nueva institución propuesta. De 1991 a 1994 el Instituto Nacional de Salud procedió estudiar y proyectar la creación de la nueva institución, creada por el Congreso de la República conforme el Artículo 45 de la Ley 100 del 23 de diciembre de 1993.

Se buscó apoyo de instituciones de orden mundial. Así, la Organización Panamericana de la Salud fue informada del proyecto de creación del nuevo organismo y manifestó su aceptación y apoyo. Igualmente, el Departamento de Salud y Servicios de Salud (HHS), Administración de Alimentos y Drogas (FDA) de los Estados Unidos de Norte América, que contribuyeron con información útil para el objetivo propuesto.

Paralelo a los esfuerzos que el Ministerio de Salud y el Instituto Nacional de Salud hacían para lograr la creación del InVIMA, se desarrollaron planes de vigilancia y control tendientes al aseguramiento de la calidad de los medicamentos, vacunas, cosméticos, alimentos, bebidas y demás productos que requerían registro sanitario, mediante la ejecución de programas de análisis y pruebas de laboratorio. De igual forma, se ejecutaron programas de vigilancia e inspección en los procesos de fabricación, supervisando el cumplimiento de las buenas prácticas de manufactura en laboratorios y fábricas.

Entre 1993 y 1994 la División de Control de Medicamentos del Instituto Nacional de Salud, en coordinación con la Subdirección de Control de Factores de Riesgo del Consumo del Ministerio de Salud y las direcciones seccionales de salud, desarrolló un plan de control de calidad sobre 500 muestras de medicamentos esenciales (Plan 500), teniendo en cuenta su volumen del riesgo de inestabilidad y contaminación, los productos de marca y los genéricos. Se buscaba verificar el cumplimiento de las especificaciones contempladas en las normas del Ministerio de Salud en relación con el etiquetado, características de empaque y el cumplimiento de las especificaciones dadas por las farmacopeas oficiales para cada medicamento y cada una de sus presentaciones.

Los resultados mostraron que $31(6,2 \%)$ de las 500 muestras analizadas, no cumplían las especificaciones de calidad de las farmacopeas oficiales. Las muestras con mayor porcentaje de rechazo provenian de productos cardiovasculares y antihipertensores, pues de 135 analizadas, 15 no cumplían con las normas (11.1\%). En el caso de productos gastrointestinales para enfermedad ácido péptica, de 62 muestras, 8 también incumplían las normas (12,9\%), poniendo en riesgo la salud de los consumidores (4).

Fueron muchas las instituciones de gran reconocimiento nacional e internacional que participaron con sus conocimientos y experiencias para diseñar una solución a esta situación; de este modo, la creación del Invima, como organismo encargado de la vigilancia y control de los alimentos y bebidas, significó un avance hacia el bienestar y salud de los ciudadanos.

El papel del InVIMa: la Ley 100 del 23 de diciembre de 1993 en su artículo 245 creó el Instituto Nacional de Vigilancia de Medicamentos y Alimentos como un establecimiento público de orden nacional, adscrito al Ministerio de Salud cuyo objeto es la ejecución de las políticas en materia de vigilancia sanitaria y de control de calidad de medicamentos, productos biológicos, alimentos, bebidas, cosméticos, dispositivos y elementos médico-quirúrgicos, odontológicos, productos naturales homeopáticos y los generados por biotecnología, reactivos de diagnóstico, y otros que puedan tener impacto en la salud individual y colectiva.

El Invima comenzó labores en febrero de 1995 con 25 funciones entre las que vale la pena resaltar:

- Controlar y vigilar la calidad y seguridad de los productos de su competencia y en las demás 
normas pertinentes, durante todas las actividades asociadas con su producción, importación, comercialización y consumo.

- Efectuar las pruebas de laboratorio que considere de mayor complejidad a los productos atrás indicados y en las demás normas pertinentes.

- Identificar, proponer y colaborar con las entidades competentes, en la investigación básica, aplicada y epidemiológica de las áreas de su competencia.

El Estado tiene la intención de acabarlo: la esencia de ser del InviMA como Institución del Estado es el bienestar de la comunidad (consumidores y productores) con el aseguramiento de la calidad de los bienes de consumo, por tanto toda acción que lleve a este fin fortalece la institución. Si el INVIMA está cumpliendo cabalmente con las funciones que se le asignaron no hay razones valederas para que el Estado manifieste sus intenciones de acabarlo y que sus funciones sean asumidas por otras Entidades entre ellas la Superintendencia de Salud y la Superintendencia de Industria y Comercio como lo anunció la presidencia de la república.

Durante el 2004, los sectores industriales se quejaron de la lentitud de los trámites, algunos de ellos innecesarios, que se llevan a cabo en el INviMA y el Presidente de la República manifestó que si el Invima no mejoraba, sería liquidado y sus funciones serían asumidas por otras dependencias estatales.

Otros sectores manifiestan insatisfacción pues para ellos el control que está ejecutando no es efectivo, siendo más de papel que de acciones de inspección y de pruebas análisis de laboratorio, y que no se cumplen los términos de Ley establecidos para los trámites.

Igualmente, se debe conocer si ha ejecutado planes de control de calidad a través de pruebas de laboratorio a los diferentes productos bajo su competencia, pues si bien es cierto que la vigilancia y control se pueden hacer por procesos de inspección verificando el cumplimiento de las buenas prácticas de manufactura, también es innegable la importancia de las pruebas de laboratorio en el proceso de la vigilancia y control.

Sería de interés conocer qué acciones ha ejecutado el Invima durante su período de vida de 10 años, en el desarrollo de otras de sus funciones como son: la Investigación básica, investigación aplicada y epidemiológica en las áreas de su competencia y, si lo ha ejecutado, donde estan publicadas.

Sin embargo, las deficiencias en la ejecución de sus funciones no implican acabarlo, por el contrario, es necesario identificar las causas y hacer las respectivas correcciones, antes de tomar decisiones que pueden llegar a causar graves daños en el sistema de vigilancia y control de los productos de consumo y retroceder así a la situación existente a mediados del siglo pasado.

El Invima debe revisar sus políticas y sus procedimientos pues lo manifestado por la Presidencia se convirtió en un campanazo de alerta.

Finalmente si las autoridades competentes tienen evidencias que demuestran que el Instituto Nacional de Vigilancia de Medicamentos y Alimentos no está en capacidad de cumplir con las funciones encomendadas es decir que fue incompetente a través de los 10 años de funcionamiento, el Estado debe actuar en forma rigurosa, aplicando los correctivos pertinentes.

El ánimo de escribir este artículo no es más que defender las razones que se tuvieron en cuenta para la creación una institución qué, como el INviMA, debe asegurar la calidad de los bienes de consumo primarios y que no merece tener una muerte anunciada.

\section{Referencias}

1. Correa FA. Codificación Nacional de Higiene. Departamento Jurídico del Ministerio de Higiene. 1949.1011 p.

2. Ortega DY, Quevedo F. Garantía de calidad de los laboratorios de microbiología alimentaria. Oficina Panamericana de la Salud. México; 1991. 152 p.

3. López AE, Barbosa E, Rodríguez G. Contenido de alcohol metílico en alcoholes antisépticos colombianos. Acta Médica del Valle 1978; 2: $80-3$

4. López AE. Informe final. Plan 500. Análisis de 500 muestras de medicamentos. Subdirección Vigilancia Sanitaria de Medicamentos y Alimentos. División Control de Medicamentos. INS; 1994. 25 p.

www.unicolmayor.edu.co 